DE

M E D I C I N A

T R O P I C A L

$\mathrm{DE}$

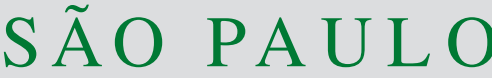

JOURNAL OF THE SÃO PAULO INSTITUTE OF TROPICAL MEDICINE

(1)Universidade Estadual de Maringá, Departamento de Análises Clínicas e Biomedicina, Laboratório de Micologia Médica, Maringá, Paraná, Brazil

(2)Universidade Federal do Paraná, Departamento de Biologia Celular, Curitiba, Paraná, Brazil

(3)Universidade Estadual de Maringá, Departamento de Análises Clínicas e Biomedicina, Laboratório de Citologia Clínica, Maringá, Paraná, Brazil

Correspondence to: Marcia Edilaine Lopes Consolaro

Universidade Estadual de Maringá, Departamento de Análises Clínicas e Biomedicina, Laboratório de Citologia Clínica, Av. Colombo, 5790, Zona 7, CEP 87020-900, Maringá, PR, Brazil Tel: +55 44 3011-4795, Fax: +55 44 30114860 .

E-mail: melconsolaro@gmail.com

Received: 3 October 2016

Accepted: 22 February 2017

\section{Virulence factors and genetic variability of vaginal Candida albicans isolates from HIV-infected women in the post-highly active antiretroviral era}

\author{
Pâmela Cristina Mastellaro Delvas Zanni ${ }^{1,3}$, Patrícia de Souza Bonfim- \\ Mendonça', Melyssa Negri', Sandra Sayuri Nakamura', Lucélia Donatti ${ }^{2}$, \\ Terezinha Inez Estivalet Svidzinski ${ }^{1}$, Márcia Edilaine Lopes Consolaro ${ }^{3}$
}

KEYWORDS: Vulvovaginal candidiasis. Candida albicans. HIV. Virulence. Genetic variability.

\section{INTRODUCTION}

Vulvovaginal candidiasis (VVC) is a disease caused by the abnormal growth of yeasts of the genus Candida in the mucosa of the female genital tract. The yeasts, in particular $C$. albicans, are well adapted to the human body and are capable of colonization with no signs of disease in conditions of physiological equilibrium ${ }^{1,2}$. However, under conditions that disrupt the delicate balance between the host and this commensal fungus, a parasitic relationship may occur, resulting in the development of infections termed candidiasis, including $\mathrm{VVC}^{2-4}$. Predisposing host factors, including immunosuppressive diseases such as HIV infection, are very important in the development of $\mathrm{VVC}^{4-6}$. The use of highly active antiretroviral therapy (HAART) has extended the life expectancy of HIV-infected individuals. However, some studies have reported that opportunistic infections remain a serious problem even in patients undergoing HAART ${ }^{5-7}$. Despite its importance, relatively little is known about the pathogenesis of VVC in HIV-infected women in the post-HAART era.

VVC is one of the most common fungal infections, frequently recurring in HIV infected women ${ }^{5}$. Although VVC is not known as an acquired immunodeficiency syndrome (AIDS)-defining condition, cases of VVC are often diagnosed among 
women with HIV-associated immunosuppression ${ }^{7}$. Symptoms of VVC are pruritus, discomfort, dyspareunia, and dysuria. Vulval infection presents as a morbilliform rash that may extend to the thighs. Vaginal infection is associated with white discharge and vaginal walls present with erythema and plaques. Although rarely or almost never resulting in systemic fungal infection or mortality, these lesions, if left untreated, contribute to the morbidity associated with HIV infection ${ }^{5}$.

C. albicans is the most common agent isolated from VVC in HIV-infected and uninfected women, appearing in $80-90 \%$ of cases in some reports ${ }^{1,6}$. The transformation from a harmless commensal to a virulent pathogen due to a dysfunctional host defense system involves an extensive repertoire of selectively expressed fungal virulence determinants ${ }^{8}$. Of these, adhesion capacity, biofilm development, cell surface hydrophobicity, morphological transition and production of hydrolytic enzymes by $C$. albicans are recognized essential steps in the process of Candida colonization and subsequent infection ${ }^{8-12}$. Genetic variability is related to $C$. albicans virulence due to the microevolution of strains within a particular species ${ }^{13}$. However, in spite of technological advances and the fact that $\mathrm{VVC}$ is a multifactorial disease, little is known on the mechanisms that make virulence factors and genetic variability contribute to the pathogenesis of vaginal $C$. albicans in HIV-infected women, so that their influence on the development of VVC remains unclear.

Here, we report a comparative analysis of key virulence factors and genetic variability of vaginal $C$. albicans from HIV-infected women receiving HAART and HIVuninfected women.

\section{MATERIALS AND METHODS}

\section{Strains}

A total of 44 vaginal C. albicans isolates were previously collected, 26 from HIV-infected women (HIV+ group) and 18 from uninfected women (HIV-group). All woman from the HIV+ group were undergoing HAART for less than 10 years, provided by the Brazilian Health Minister and the Specialized Assistance Service for Sexually Transmitted Disease (SAS-STD)/AIDS in Maringá, Brazil. The therapeutic regimen adopted was the combination of at least an antiviral targeting the HIV protease. C. albicans isolates were separated into two groups according to clinical signs and symptoms presented by the women: colonization (COL) in cases with positive vaginal culture but no symptoms; and infection (INF) in those cases with positive vaginal culture and at least two symptoms of
VVC (vaginal discharge, burning, itching, dysuria and/ or dyspareunia) $)^{14}$. All clinical isolates were identified by classical and biochemical methods ${ }^{15}$ confirmed by matrixassisted laser-desorption/ionization time-of-flight mass spectroscopy (MALDI TOF-MS). For MALDI TOF-MS, the yeasts were prepared and analyzed according to previous reports ${ }^{16,17}$ with a Microflex LT mass spectrometer (Bruker Daltonics) using the FlexControl software (version 3.0, Bruker Daltonics). The yeasts were stored in the culture collection of the Medical Mycology Laboratory of the State University of Maringá (UEM), Brazil. For each experiment, C. albicans isolates and C. albicans (ATCC 90028) from the American Type Culture Collection were subcultured on Sabouraud dextrose agar (SDA; Difco, Becton, Dickinson and Company, Sparks, Maryland, USA) overnight at $37^{\circ} \mathrm{C}$. All virulence factors studied were assessed in triplicate and in three independent assays.

\section{Proteinase activity assay}

Proteinase activity was measured according to a previous report by Kuriyama et al. ${ }^{18}$, with modifications. C. albicans isolates were subcultured in yeast extract peptone dextrose broth (YEPD - Becton, Dickinson and Company, Sparks, Maryland, USA) at $37{ }^{\circ} \mathrm{C}$ for $24 \mathrm{~h}$. The culture was washed, the concentration was adjusted to $1 \times 10^{6}$ yeast/ $\mathrm{mL}$, and $500 \mu \mathrm{l}$ of the suspension was added to $20 \mathrm{~mL}$ of yeast carbon base plus bovine serum albumin medium (YCB-BSA; Becton Dickinson, Oxford, UK) followed by incubation in a shaker at $37{ }^{\circ} \mathrm{C}$ for $48 \mathrm{~h}$. The yeast concentrations were determined and then the solution was centrifuged. A volume $(100 \mu \mathrm{L})$ of YCB-BSA culture supernatant was mixed with $400 \mu \mathrm{L}$ of $0.1 \mathrm{M}$ sodium citrate buffer (LabSynth, Diadema, São Paulo, Brazil) containing 1\% bovine hemoglobin (Sigma, St. Louis, Missouri, USA). This mixture was incubated at $37^{\circ} \mathrm{C}$ for $1 \mathrm{~h}$. After incubation, $500 \mu \mathrm{L}$ of $20 \%$ trichloroacetic acid (TCA; Sigma, St. Louis, Missouri, USA) were added and the mixture was incubated on ice for $1 \mathrm{~h}$ to precipitate any undigested protein. The precipitate was centrifuged and the peptide concentration from the supernatant was measured in a Nanodrop spectrophotometer (NanoDrop 2000 UV-Vis Spectrophotometer, Thermo Scientific, Wilmington, Delaware, USA) at $280 \mathrm{~nm}$. The control samples were inoculated into substrate broths that were immediately treated with TCA, without the $1 \mathrm{~h}$ incubation period. Proteinase activity was calculated based on the absorbance differences between samples and controls. One proteinase activity unit was defined as a 0.1 increase in the optical density (OD) at $280 \mathrm{~nm} / \mathrm{h}$. This value was related to the initial yeast $/ \mathrm{mL}$ concentration to calculate the final 
proteinase activity ${ }^{11}$. To confirm the proteinase activity, the culture supernatants were mixed with hemoglobin-sodium citrate buffer containing $50 \mathrm{mg} / \mathrm{mL}$ pepstatin A (Sigma, St. Louis, Missouri, USA).

\section{Cell surface hydrophobicity (CSH) assay}

CSH was determined by testing microbial adhesion to hydrocarbons (MATH) according to Raut et al. ${ }^{12}$. C. albicans isolates were grown in YPD (Becton Dickinson, Oxford, UK) and resuspended in PBS to obtain a suspension with a final optical density (OD) of 0.5 at $620 \mathrm{~nm}$ evaluated by spectrometry (Quimis, Spectrophotometer Q7980RM, Diadema, SP, Brazil). A total of $100 \mu \mathrm{L}$ of this suspension was distributed into 96-well microplates. The plate was read at $620 \mathrm{~nm}$ using a microplate reader (Asys High Tech Expert Plus UV, Austria) and this value was designated as the initial OD of the yeast suspension. After the reading, $300 \mu \mathrm{L}$ of n-octane were added to the wells, the samples were shaken for $3 \mathrm{~min}$., and the plate was allowed to stand for $15 \mathrm{~min}$. to separate the phases. Subsequently, $10 \mu \mathrm{L}$ of each lower aqueous phase was carefully removed and allocated to a new microplate well, and the final OD readings were obtained under the same conditions. The CSH percentage is given by the formula: \% CSH $=(1-\mathrm{OD}$ of the final aqueous phase/OD of the initial yeast suspension) x 100 .

\section{Adhesion and biofilm formation assays}

The assays were performed according to Negri et al. ${ }^{19}$. Yeast cells were grown at $37{ }^{\circ} \mathrm{C}$ in a shaker at $120 \mathrm{rev} / \mathrm{min}$ for $18 \mathrm{~h}$ in Sabouraud dextrose broth (SDB; Difco, Becton, Dickinson and Company, Sparks, Maryland, USA). Yeast cells were washed with phosphate-buffer saline (PBS) and resuspended in RPMI 1640 (Sigma, Saint Louis, Missouri, USA) to a final concentration of $1 \times 10^{7}$ yeast $/ \mathrm{mL}$. Then, $200 \mu \mathrm{L}$ of suspension was added to each well of a 96-well plate and incubated for $2 \mathrm{~h}$ for adhesion assays or $24 \mathrm{~h}$ for biofilm formation assays. Yeast cell adhesion and biofilm formation were quantified using the crystal violet staining method.

\section{Scanning electron microscopy (SEM)}

To examine yeast cell adhesion by SEM, $2 \mathrm{~mL}$ of the standardized cell suspension $\left(1 \times 10^{7}\right.$ yeast $/ \mathrm{mL}$ in RPMI 1640) was added to the wells of 24 -well polystyrene plate and incubated for $2 \mathrm{~h}$ at $37^{\circ} \mathrm{C}$ in a shaker at $120 \mathrm{rev} / \mathrm{min}$. After $2 \mathrm{~h}$, the medium was aspirated, and non-adherent cells were removed by washing twice with PBS. Samples were fixed with $2.5 \%$ glutaraldehyde diluted in $0.1 \mathrm{M}$ cacodylate buffer (Sigma, St. Louis, Missouri, USA) and dehydrated with an ascending series of alcohol solutions. Samples were kept in a desiccator until the bases of the wells were removed for analysis. Prior to observation, the bases of the wells were mounted onto aluminum stubs, sputter coated with gold and observed with a JEOL-JSM 6360 LV scanning electron microscope (Jeol Ltd., Tokyo, Japan) at the Electron Microscopy Center, State University of Paraná, Maringá, Brazil.

\section{Germ tube formation and length}

An inoculum was prepared in PBS according to the $\mathrm{N}^{\mathrm{o}} 4 \mathrm{McFarland}$ turbidity standard. This suspension was centrifuged at $1,170 \mathrm{~g}$ for $10 \mathrm{~min}$. Then, the pellet was resuspended in RPMI 1640 medium containing fresh heat-inactivated human serum (1:1) and incubated for $3 \mathrm{~h}$ at $37{ }^{\circ} \mathrm{C}$. The germ tubes were defined according to the description of Hammer et al. ${ }^{20}$ as a cell bearing a rounded outgrowth with a length greater than or equal to the diameter of the parent cell, not constricted at the base. Germ tubes were counted and measured with a light microscope (Nikon, TMD; Nippon Kogaku Inc.) equipped with a calibrated eyepiece graticule. Their frequency and length were determined in a blinded manner in 200 cells from each of the samples. The results were expressed as the average \pm standard deviation (SD).

\section{RAPD analysis}

C. albicans isolates were analyzed according to Bonfim et al. ${ }^{21}$ using primers OPA-18 (5'-AGCTGACCGT-3') and OPE-18 (5'-GGACTGCAGA-3' ${ }^{22}$. The RAPD profiles were analyzed using the Bionumerics software version 4.6 (Applied Maths, Carlsbad, CA, USA). For the determination of the size of fragments, we used a 100 bp molecular marker (Invitrogen, São Paulo, Brazil). The similarity was verified by the coefficient $\left(S_{A B}\right)$ between each pair of standards for $\mathrm{A}$ and $\mathrm{B}$ isolates and was calculated with the formula $S_{A B}=2 E /(2 E+a+b)$, where $E$ is the number of common bands in the patterns A and $\mathrm{B} ; a$ is the number of bands with an $A$ pattern and no correlated $B$ patterns and $b$ is the number of bands with a $B$ pattern and no correlated $A$ patterns. Based on the similarity matrix, the units were grouped using an unweighted pair-group method with arithmetical average (UPGMA). An $S_{A B}$ value of 100 indicates that the pattern of bands for line $\mathrm{A}$ is identical to $\mathrm{B}$; values between 80 and 99 represent very similar but not identical clinical isolates, and may suggest microevolution of a single strain; $S_{A B}$ values less than 80 represent independent lines ${ }^{23}$. 


\section{Statistical analysis}

Data distributions were expressed as the mean \pm SD of three independent experiments, in triplicate. Significant differences among means were identified using the GraphPad Prism ${ }^{\circledR} 5.0$ software and the Mann-Whitney and Bonferroni tests. Values of $P<.05$ were considered significant.

\section{RESULTS}

\section{Antifungal susceptibility profile}

Our previous study investigated antifungal activity in vitro for Fluconazole (FLU), Itraconazole (ITRA), Nystatin (NYST) and Amphotericin B, regarding all clinical isolates used in the present study. In general, $C$. albicans isolates showed no resistance to the antifungal agents tested. For the HIV+ group, 2 (7.7\%) clinical isolates presented a dosedependent susceptibility (DDS) to FLU, 1 (3.9\%) isolate to ITRA and 1 (3.9\%) isolate to NYST. For the HIV-group, 7 (38.9\%) clinical isolates of $C$. albicans were DDS to NYST and $1(5.5 \%)$ isolate to ITRA ${ }^{24}$.

\section{Proteinase activity}

C. albicans isolates from the HIV+ group showed higher proteinase activity than $C$. albicans from the HIV- group $(P<.0001)$ (Figure 1A). Furthermore, comparing the clinical groups (Figure 1B), C. albicans from the HIV+ INF group showed significantly greater proteinase activity than $C$. albicans from the HIV- INF group $(P<.0001)$, and this activity was higher than 0.1 unit of proteinase activity $/ 10^{6}$ cells $/ \mathrm{mL}$.

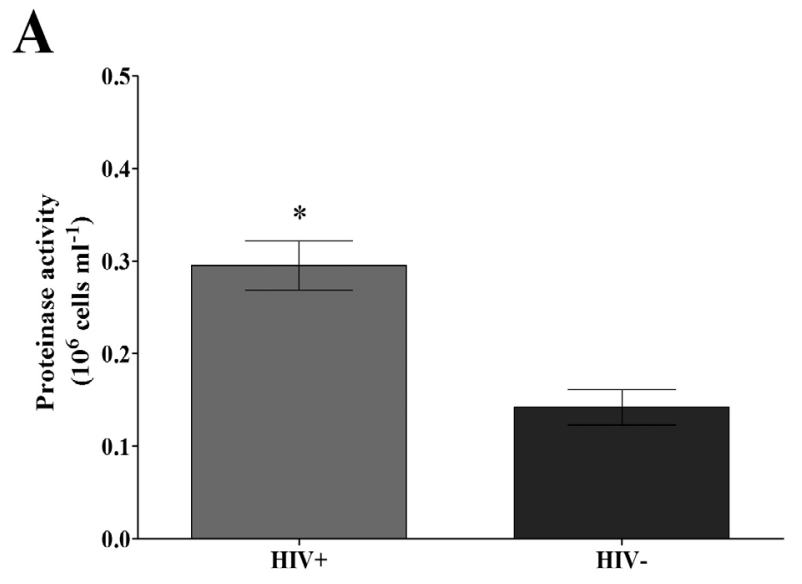

$\mathrm{CSH}$, adhesion capacity and biofilm formation

The clinical isolates showed similar $\mathrm{CSH}$; this result was independent of HIV and clinical status (Figures 2A and 2B). According to the adhesion capacity and biofilm formation assays, all isolates demonstrated these abilities; however, in general, C. albicans from both groups were more efficient in the adhesion process than in biofilm formation (Figure 3). C. albicans isolates from the HIV+ group had lower capacities to adhere and to form biofilms than $C$. albicans isolates from the HIV- group $(P<0.0001)$ (Figures $3 \mathrm{~A}$ and $3 \mathrm{C})$. In regard to the adhesion capacity in the clinical groups, yeasts from the HIV- COL (2.21 to $3.03 \mathrm{abs} / \mathrm{cm}^{2}$ ) and INF $\left(0.8\right.$ to $\left.8.59 \mathrm{abs} / \mathrm{cm}^{2}\right)$ groups showed higher adhesion values than yeasts from the HIV+ COL $(0.71$ to $\left.2.76 \mathrm{abs} / \mathrm{cm}^{2}\right)$ and INF (1.07 to $\left.3.56 \mathrm{abs} / \mathrm{cm}^{2}\right)$ groups with $P<0.0001$ (Figure 3B). C. albicans from HIV- COL (1.43 to $\left.1.82 \mathrm{abs} / \mathrm{cm}^{2}\right)$ and INF ( 0.252 to $\left.2.66 \mathrm{abs} / \mathrm{cm}^{2}\right)$ groups showed higher biofilm formation than yeasts from the HIV+ COL (0.16 to $\left.0.99 \mathrm{abs} / \mathrm{cm}^{2}\right)$ and INF (1.11 to $0.73 \mathrm{abs} / \mathrm{cm}^{2}$ ) groups with $P<0.0001$ (Figure 3B).

Based on SEM, no morphological differences were observed in the adherence of $C$. albicans to the polystyrene microplate surface among isolates from the HIV+ and HIVgroups. In the HIV+ group, blastoconidia were attached to the microplate surface, isolated or in small clusters (Figures 4A and 4B). In the HIV- group, blastoconidia attached to the surface were isolated or in large clusters (Figure 4C and 4D). Initial extracellular matrix material was noted near the blastoconidia or with embedded microorganisms (Figures 4B and 4C). We also observed budding yeasts (Figures 4B and 4D).

\section{B}

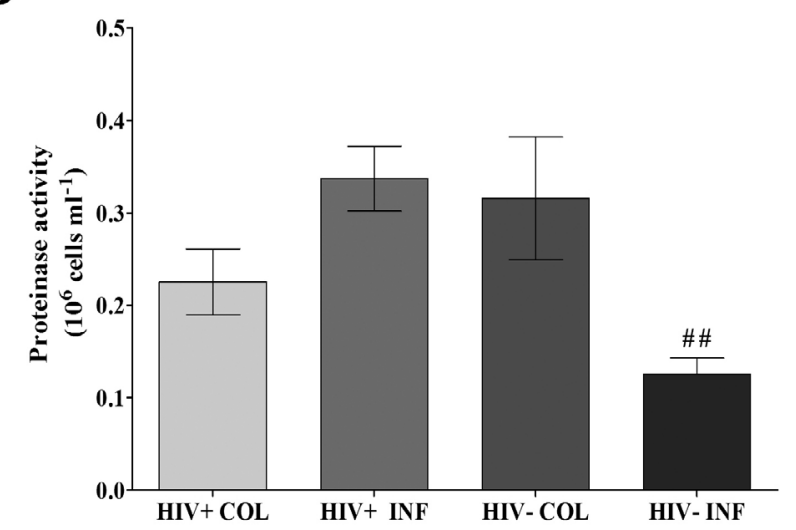

Figure 1 - Proteinase activity $\left(10^{6}\right.$ cells/ $\left.\mathrm{mL}\right)$ of vaginal Candida albicans isolated from HIV positive (HIV+) and negative (HIV-) women overall and in clinical groups: A) Proteinase activity in HIV+ and HIV- groups; B) Proteinase activity in HIV+ and HIV- groups with colonization (COL) and infection (INF). Each bar represents the mean \pm SD of the results from all isolates in each group (- three different experiments with samples tested in triplicate), ${ }^{*} p<0.05$ significant difference between $\mathrm{HIV}+$ and HIV groups. \#\#p<0.05 significant difference between HIV+INF and HIV-INF 
A

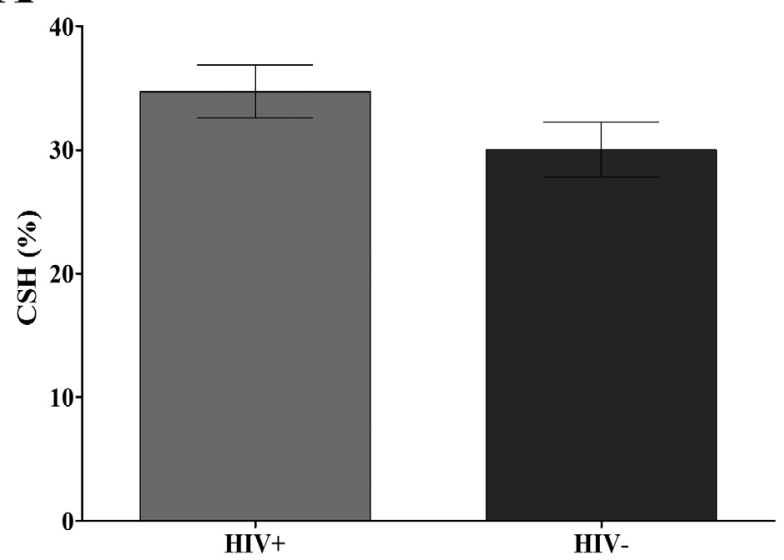

B

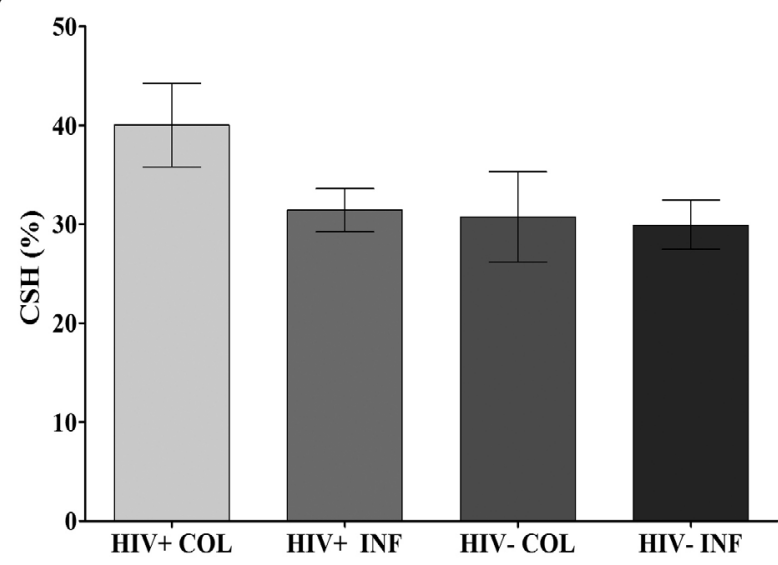

Figure 2 - Cell surface hydrophobicity (CSH) of vaginal Candida albicans isolated from HIV positive (HIV+) and negative (HIV-) women overall and in clinical groups: A) CSH (\%) in HIV+ and HIV-groups; B) CSH (\%) HIV+ and HIV-groups with colonization (COL) and infection (INF). Each bar represents the mean \pm SD of the results from all isolates in each group (three different experiments with samples tested in triplicate).

A

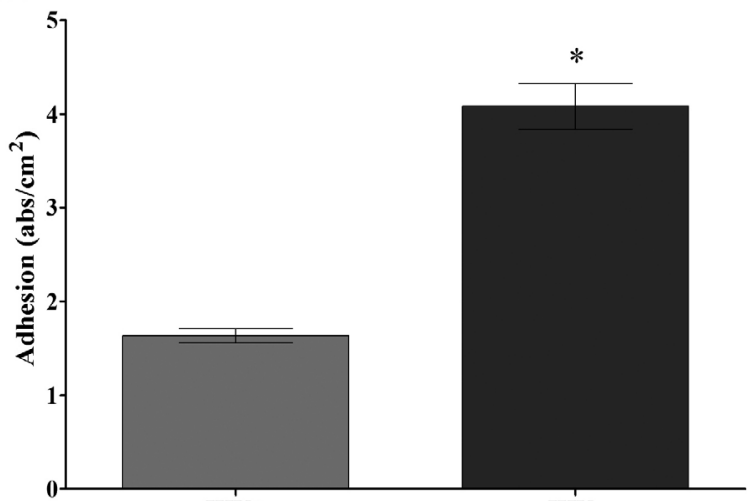

HIV+

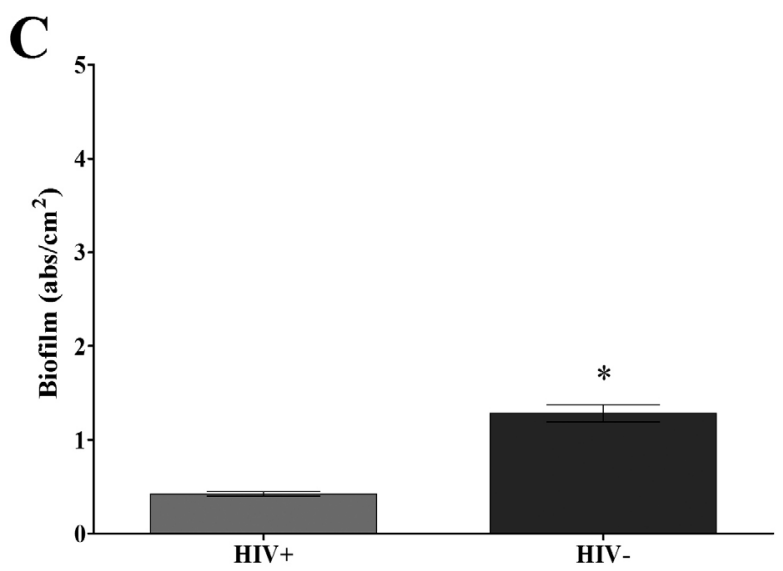

B

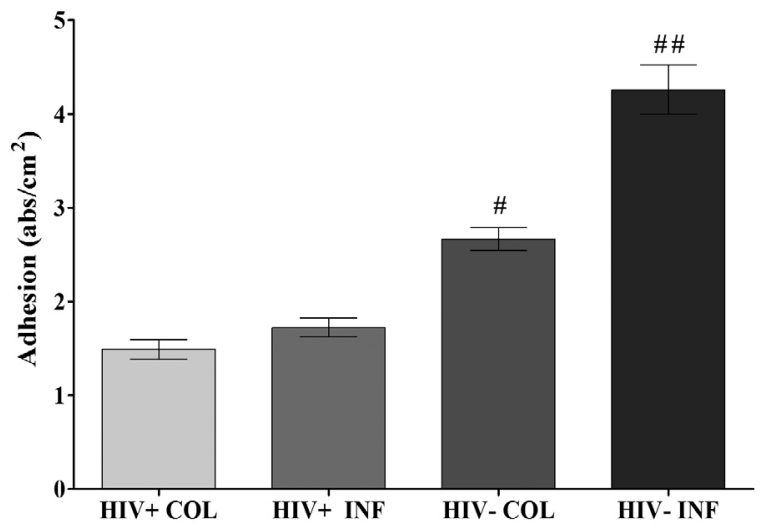

D

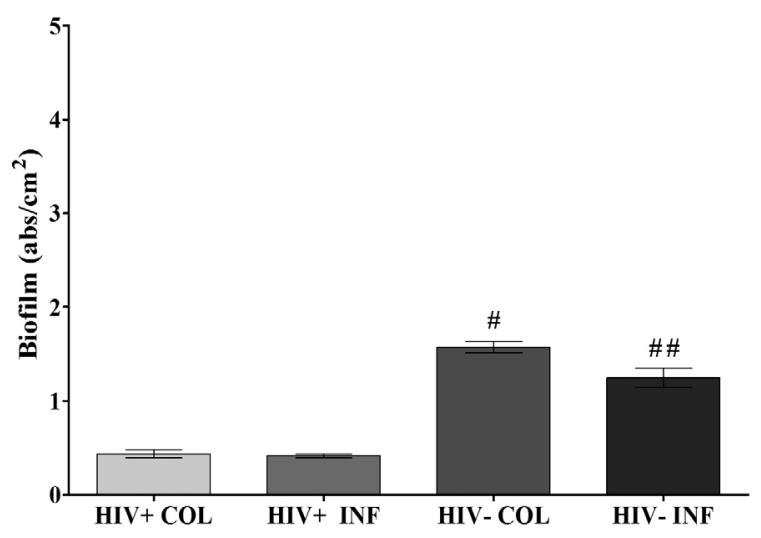

Figure 3 - Adhesion and biofilm formation capacity (absorbance $/ \mathrm{cm}^{2}$ ) of vaginal Candida albicans isolated from HIV positive (HIV+) and negative (HIV-) women overall and in clinical groups: A) Adhesion capacity in HIV+ and HIV- groups; B) Adhesion capacity in HIV+ and HIV- groups with colonization (COL) and infection (INF); C) Biofilm formation in HIV+ and HIV- groups; D) Biofilm formation in HIV+ and HIV- groups with colonization (COL) and infection (INF). Each bar represents the mean \pm SD of the results from all isolates in each group (three different experiments with samples tested in triplicate), ${ }^{*} p<0.05$ significant difference between $\mathrm{HIV}+$ and HIV- groups. \#p<0.05 significant difference between HIV+COL and HIV-COL. \#\#p<0.05 significant difference between HIV+INF and HIV-INF. 

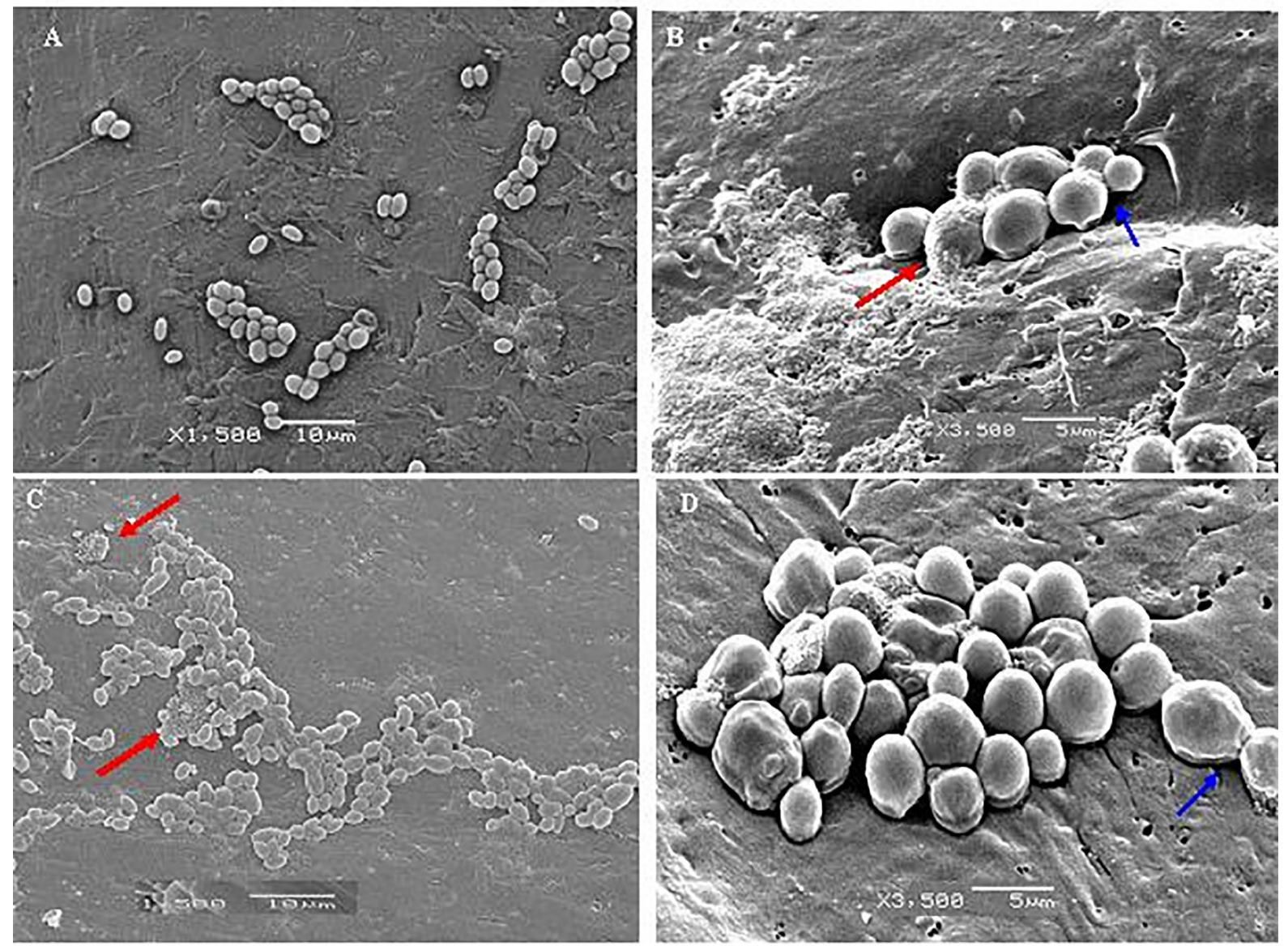

Figure 4 - Scanning electron photomicrographs showing vaginal Candida albicans from the HIV+ and HIV- groups adhered to the microplate surface after $2 \mathrm{~h}$ of contact: A) and B) HIV+ group adhesion showing yeasts in the blastoconidia form attached to the surface, isolated or in small clusters; C) and D) HIV- group adhesion showing several yeasts in the blastoconidia form attached to the surface, isolated or in large clusters. In A) and C) initial extracellular matrix material (red arrow) observed close to the blastoconidia or with microorganisms embedded. In B) and D) budding yeasts (blue arrows). Scale bars: A) and C), $10 \mu \mathrm{m} ; \mathrm{B})$ and D), $5 \mu \mathrm{m}$.

\section{Germ tube formation capacity and length}

C. albicans from the HIV- group demonstrated a higher germ tube formation capacity than $C$. albicans from the $\operatorname{HIV}+$ group $(P=.024)$ (Figure 5A). In the clinical groups, yeasts from the HIV- INF group showed a higher germ tube formation capacity than the HIV+ group $(P=.0195)$ (Figure 5B). In contrast, the germ tube length was similar between the HIV + and HIV- groups and between the clinical groups (Figures 5C and 5D).

\section{Clinical isolate similarity by RAPD}

The OPA-18 primer showed higher discriminatory power compared to the OPE-18 primer for all vaginal $C$. albicans isolates from the HIV+ and HIV- groups. For the HIV+ group, the OPA-18 primer identified 25 profiles with $\mathrm{S}_{\mathrm{AB}}$ values of $64.70 \pm 18.12$ (Figure $6 \mathrm{~A}$ ). Two groups (I and II) were formed with $36.6 \%$ similarity between them. Group I clustered $88.46 \%$ of the isolates with $66.6 \%$ similarity. Group II clustered $11.54 \%$ of isolates with $45 \%$ similarity. However, the OPE-18 primer only generated 7 different profiles, with $S_{A B}$ values of $80.95 \pm 18.71$. The analysis clustered $92.2 \%$ of the isolates with approximately $80.8 \%$ similarity (Figure 6B). For the HIV- group, primer OPA-18 identified 25 profiles with $S_{A B}$ values of $75.11 \pm 9.97$ (Figure 6C). Two groups (I and II) were formed with $36.6 \%$ similarity between them. Group I clustered $88.46 \%$ of the isolates with $66.6 \%$ similarity. Group II clustered $11.54 \%$ of isolates with $45 \%$ similarity. The OPE-18 primer only generated 7 different profiles, with a $\mathrm{S}_{\mathrm{AB}}$ value of $77.03 \pm 16.53$. The analysis clustered $92.2 \%$ of isolates with approximately $80.8 \%$ similarity (Figure 6D).

\section{DISCUSSION}

This study demonstrated that isolates of $C$. albicans from HIV+ women receiving HAART showed lower expression of virulence factors (except proteinase activity) than C. albicans from HIV- women and that expression of 
A

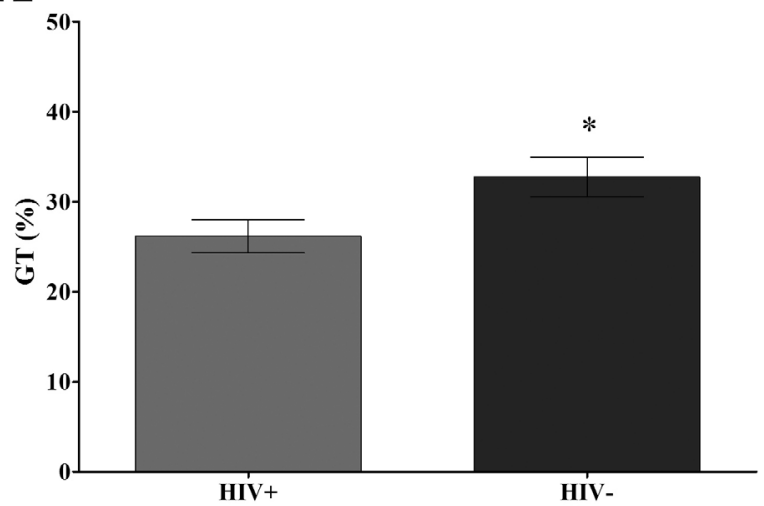

C

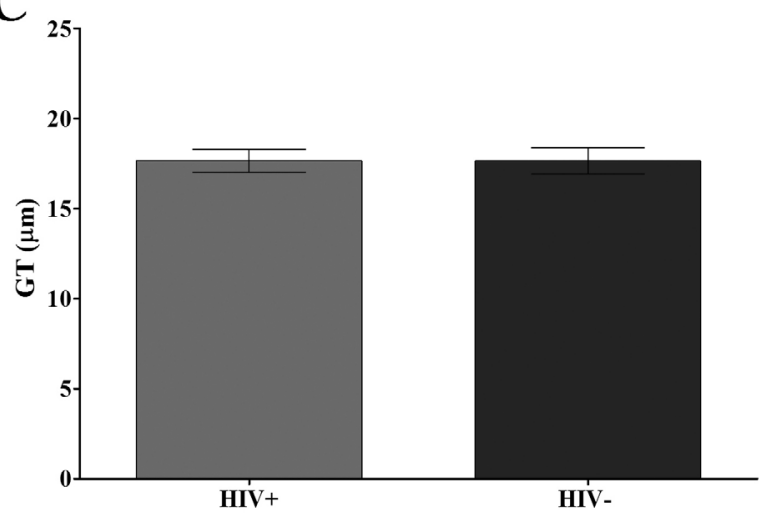

B

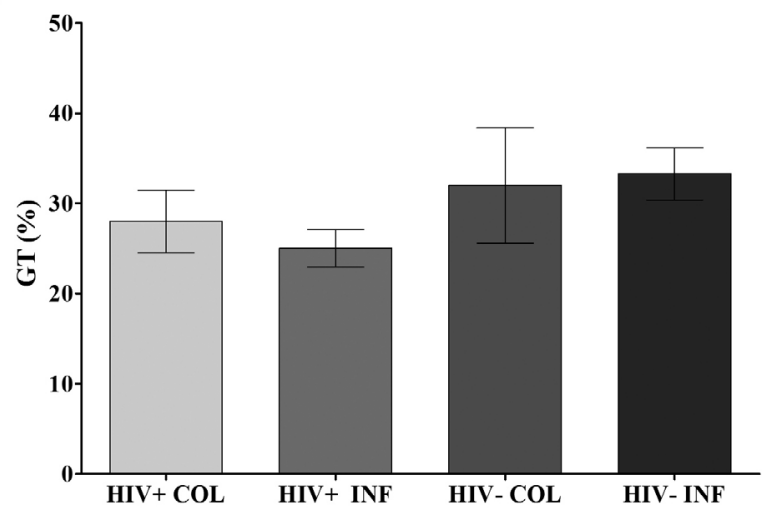

D

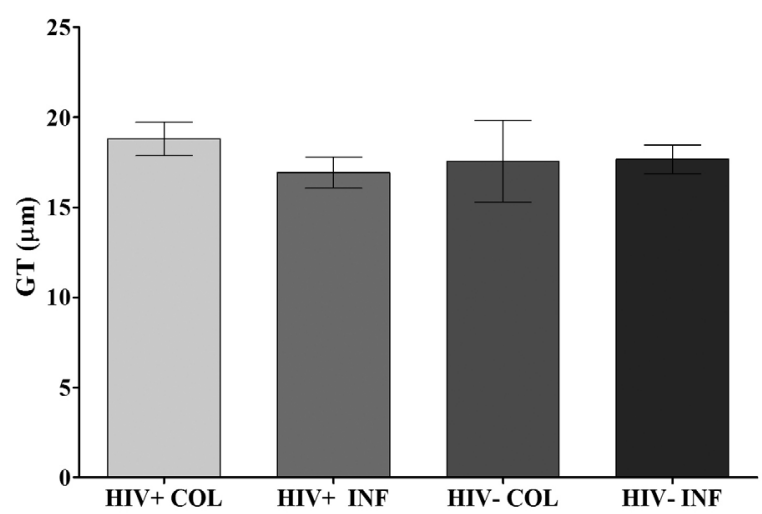

Figure 5 - Germ tube (GT) formation capacity (\%) and length $(\mu \mathrm{m})$ of vaginal Candida albicans isolated from HIV + and HIV- women overall and in clinical groups: A) GT (\%) in HIV+ and HIV- groups; B) GT in HIV+ and HIV- groups with colonization (COL) and infection (INF); C) GT length in HIV+ and HIV- groups; D) HIV+ and HIV- groups with colonization (COL) and infection (INF). Each bar represents the mean \pm SD of the results from all isolates in each group (three different experiments with samples tested in triplicate). ${ }^{*} p<0.05$ significant difference between HIV + and HIV-groups.

virulence factors was a yeast response to stress. Previous studies conducted in HIV+ women receiving HAART have indicated that vaginal colonization by yeasts is significantly increased in HIV+ women and is correlated with the loss of immunoprotective mechanisms ${ }^{25,26}$. However, little is known about the virulence factors of vaginal $C$. albicans from HIV+ women in the post-HAART era. To our knowledge, this is the first study describing these aspects comparing yeasts from HIV + and HIV- women.

The virulence factors of $C$. albicans have been defined as mechanisms that interact directly with host componentscontributing to the adherence, penetration and invasion of host tissues ${ }^{9-12}$. Proteinases are hydrolytic enzymes that contribute to the process of virulence and play an important role in the pathogenesis of $\mathrm{VVC}^{27}$. In this study, C. albicans from HIV+ women showed significantly higher proteinase activity than yeasts from HIV- women (Figure 1A). Similar results were found by De Bernardis et al. ${ }^{28}$ in C. albicans isolates from the oral cavity of HIV+ women showing increased proteinase activity compared with isolates from HIV- women. Although some reports have suggested that patients undergoing HAART with protease inhibitors (PI) show decreased proteinase activity of $C$. albicans ${ }^{29-31}$, our results showed the opposite, C. albicans isolates from HIV+ women had increased proteinase activity, and isolates from the HIV+/INF group showed two-fold greater proteinase activity than $C$. albicans from the HIV-/INF group. These findings may explain, at least partially, why opportunistic VVC infections remain a serious problem even in patients under HAART treatment ${ }^{5-7}$. However, these results did not clarify whether HAART with PI treatment had a persistent effect on fungal infection or carriage in vaginal mucosa. It has been suggested that proteinase production is a highly regulated process that is likely to reflect the complex transcriptional co-regulation with other virulence factors of $C$. albicans. The combination of virulence factors may promote the pathogenicity of C. albicans ${ }^{9,27}$.

CSH contributes to interactions between cells and adjacent surfaces and seems to be an important factor in the adherence of $C$. albicans to inert surfaces ${ }^{12,32}$. Our results showed similar CSH in yeasts from HIV+ and HIV- women, irrespective of clinical groups. It has been observed that 

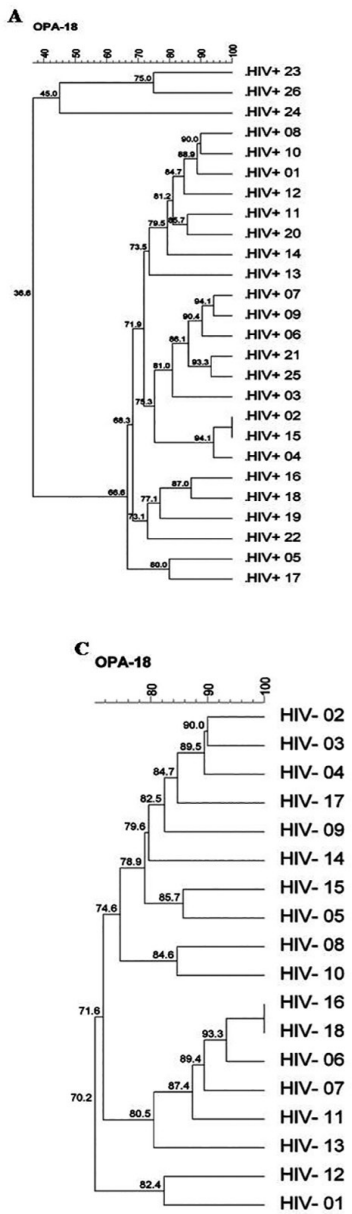
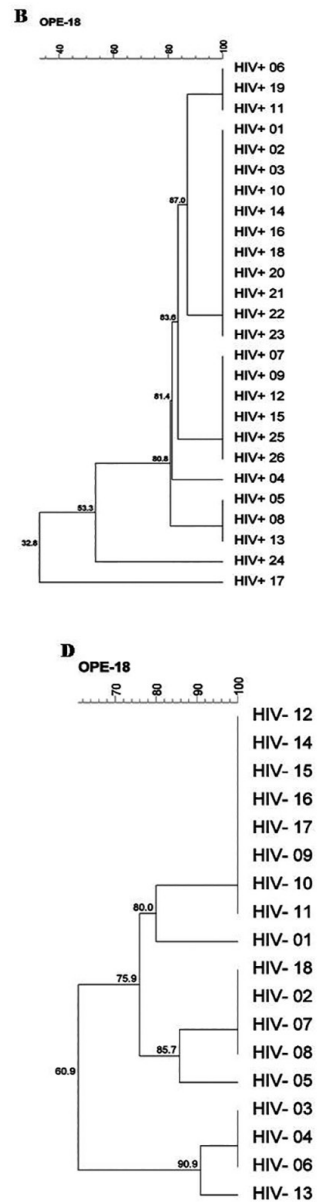

Figure 6 - Dendrogram generated by RAPD analysis of vaginal Candida albicans isolated from HIV + and HIV- women using the UPGMA (unweighted pair-group method with arithmetical average) grouping, with $S_{A B}$ calculated by the coefficient of Dice: A) and C) Yeast dendrograms using the OPA-18 primer; B) and D) Yeast dendrograms were constructed using the OPE18 primer results. The numbers indicated on the right side of dendrograms represent the registration of each yeast in the culture collection of the Medical Mycology Laboratory of the State University of Paraná, Maringá (UEM), Brazil.

hydrophobic molecules are expressed to facilitate a stronger adhesion $^{33,34}$. However, other important factors, such as the surface properties of both microorganisms and materials, as well as environmental factors, also increase or decrease the adhesion capacity $3,9,11,32$.

C. albicans from HIV+ women had a lower capacity for adhesion and biofilm formation than C. albicans from HIV- women, in both clinical groups (Figures 3A and 3C). Adhesion generally involves the formation of blastoconidia, the occasional formation of hyphae and an initial production of extracellular matrix, which was observed by SEM in this study, confirming results observed in other studies ${ }^{35}$. However, the incubation period is a critical factor, and prolonged periods of time allow for the formation of complex biofilms, with blastoconidia and hyphae-like forms, as demonstrated in C. albicans by Paiva et al. ${ }^{35}$.

While adhesion capacity is important for tissue infection, biofilm formation is an important process that helps to maintain the yeast on natural host surfaces ${ }^{10}$. When Candida forms biofilms, it displays phenotypic properties that are different from those in planktonic growth, such as enhanced resistance to antifungals and neutrophils and altered gene expression, allowing the yeast to remain in a hostile environment where it is often in competition with other microorganisms, in addition to the local response of the host ${ }^{8,10}$. Mane et al. $^{8}$ showed results that differed from ours in yeast isolated from the oral cavity of HIV+ individuals; the yeast showed increased biofilm formation. This is an important difference of yeasts from the vaginal mucosa of HIV+ women and deserves further study. Furthermore, oral environments promote increased development of biofilms due to the variability of oral hygiene, salivation and greater variability of the local microbiota ${ }^{36}$.

Both blastoconidia and hyphal cells of $C$. albicans are found in infected host tissues, and it is widely believed that hyphal formation is important for its invasive properties and is associated with adhesion and invasion of tissues ${ }^{12}$. However, our results showed less germ tube formation in C. albicans isolates from HIV+ compared to HIV- women, although the germ tube length was similar in both groups. These transitions between yeast, pseudohyphal and hyphal growth forms contribute to the virulence of $C$. albicans, which interacts directly with host components as a response to stress $^{25,26}$. These results suggest that strains submitted to lower levels of stress had attenuated virulence factors and strains that suffered greater stress had enhanced pathogenicity against the host. According to Brown et al. ${ }^{25}$, stress leads to an adaptive response of the host although it is not clear whether this genetic adaptation is observed both in vivo and in vitro.

Clustering analysis using the RAPD method showed that, in general, the isolates from HIV+ and HIV- women showed a high degree of heterogeneity. The range of mean $S_{A B}$ values was less than $80 \%$ in all the primers examined, suggesting the presence of independent strains and confirming the results of Chong et al. ${ }^{23}$. Low similarity among vaginal isolates has been reported by other authors ${ }^{37}$. No correlation was observed between the clustering and virulence factors analyzed. These results demonstrate that vaginal colonization or $\mathrm{VVC}$ varies among individuals and ranges between strain maintenance, strain microevolution and strain replacement; the major scenario is the strain maintenance with microevolution ${ }^{23}$.

The results of this study suggest that $C$. albicans virulence factors from HIV+ and HIV- individuals are expressed in response to yeast stress. Recent studies 
indicated that heterogeneous behaviors in specific host niches lead to different genetic adaptations ${ }^{25}$. Moreover, virulence factors should never have been considered independent of the host's defenses, and the clinical course of a disease often depends on the interaction of virulence factors with the host's response. It is known that the integrity of the immune system (physical and chemical attributes) is able to stimulate the virulence of the microorganisms ${ }^{38}$. Additionally, previous results from our group ${ }^{24}$ showed that clinical isolates from both groups-did not have an antifungal resistance profile, suggesting that the presence of yeast in the vaginal environment of the studied women is in fact related to virulence factors.

In summary, our study demonstrated that isolates of C. albicans from HIV+ women undergoing HAART had lower expression of virulence factors than isolates from HIV- women, with the exception of proteinases, which were highly expressed. The study further demonstrated that virulence factors are expressed according to the level of stress to which the yeast was exposed. These findings will allow the better monitoring of vaginal yeasts infections in HIV-infected women under HAART treatment which will improve the quality of life of patients.

\section{ACKNOWLEDGMENTS}

This work was supported by Coordenação de Aperfeiçoamento de Pessoal de Nível Superior (CAPES), Brazilian Government Grant [AUX-PE-PRODOC 2571/2010].

\section{CONFLICT OF INTERESTS}

The authors report no conflicts of interest. The authors alone are responsible for the content and the writing of the paper.

\section{REFERENCES}

1. Sobel JD. Vulvovaginal candidosis. Lancet. 2007;369:1961-71.

2. Hamad M, Kazandji N, Awadallah S, Allam H. Prevalence and epidemiological characteristics of vaginal candidiasis in the UAE. Mycoses. 2014;57:184-90.

3. Mayer FL, Wilson D, Hube B. Candida albicans pathogenicity mechanisms. Virulence. 2013;4:119-28.

4. Richardson JP, Moyes DL. Adaptive immune responses to Candida albicans infection. Virulence. 2015;6:327-37.

5. Ray A, Ray S, George AT, Swaminathan N. Interventions for prevention and treatment of vulvovaginal candidiasis in women with HIV infection. Cochrane Database Syst Rev. 2011;CD008739.
6. Merenstein D, Hu H, Wang C, Hamilton P, Blackmon M, Chen H, et al. Colonization by Candida species of the oral and vaginal mucosa in HIV-infected and noninfected women. AIDS Res Hum Retroviruses. 2013;29:30-4.

7. Apalata T, Longo-Mbenza B, Sturm A, Carr W, Moodley P. Factors associated with symptomatic vulvovaginal candidiasis: a study among women attending a primary healthcare clinic in Kwazulu-Natal, South Africa. Ann Med Health Sci Res. 2014;4:410-6.

8. Mane A, Kulkarni A, Risbud A. Biofilm production in oral Candida isolates from HIV-positive individuals from Pune, India. Mycoses. 2013;56:182-6.

9. Naglik JR, Challacombe SJ, Hube B. Candida albicans secreted aspartyl proteinases in virulence and pathogenesis. Microbiol Mol Biol Rev. 2003;67:400-28.

10. Ramage G, Saville SP, Thomas DP, López-Ribot JL. Candida biofilms: an update. Eukaryot Cell. 2005;4:633-8.

11. da Costa KR, Ferreira JC, Lavrador MA, Baruffi MD, Candido RC. Virulence attributes and genetic variability of oral Candida albicans and Candida tropicalis isolates. Mycoses. 2012;55:e97-e105.

12. Raut J, Rathod V, Karuppayil SM. Cell surface hydrophobicity and adhesion: a study on fifty clinical isolates of Candida albicans. Nihon Ishinkin Gakkai Zasshi. 2010;51:131-6.

13. Enger L, Joly S, Pujol C, Simonson P, Pfaller M, Soll DR. Cloning and characterization of a complex DNA fingerprinting probe for Candida parapsilosis. J Clin Microbiol. 2001;39:658-69.

14. Lopes Consolaro ME, Albertoni TA, Yoshida CS, Mazucheli J, Peralta RM, Svidzinski TI. Correlation of Candida species and symptoms among patients with vulvovaginal candidiasis in Maringá, Paraná, Brazil. Rev Iberoam Micol. 2004;21:2025.

15. Larone DH. Medically important fungi: a guide to identification. $5^{\text {th }}$ ed. Washington D.C.: ASM; 2011.

16. Spanu T, Posteraro B, Fiori B, D’Inzeo T, Campoli S, Ruggeri A, et al. Direct maldi-tof mass spectrometry assay of blood culture broths for rapid identification of Candida species causing bloodstream infections: an observational study in two large microbiology laboratories. J Clin Microbiol. 2012;50:176-9.

17. Pascon RC, Bergamo RF, Spinelli RX, de Souza ED, Assis DM, Juliano L, et al. Amylolytic microorganism from Sao Paulo zoo composting: isolation, identification, and amylase production. Enzyme Res. 2011;2011:679624.

18. Kuriyama T, Williams DW, Lewis MA. In vitro secreted aspartyl proteinase activity of Candida albicans isolated from oral diseases and healthy oral cavities. Oral Microbiol Immunol. 2003;18:405-7.

19. Negri M, Gonçalves V, Silva S, Henriques M, Azeredo J, Oliveira R. Crystal violet staining to quantify Candida adhesion to epithelial cells. Br J Biomed Sci. 2010;67: 120-5. 
20. Hammer KA, Carson CF, Riley TV. Melaleuca alternifolia (tea tree) oil inhibits germ tube formation by Candida albicans. Med Mycol. 2000;38:355-62.

21. Bonfim-Mendonça PS, Fiorini A, Shinobu-Mesquita CS, Baeza LC, Fernandez MA, Svidzinski TI. Molecular typing of Candida albicans isolates from hospitalized patients. Rev Inst Med Trop Sao Paulo. 2013;55:385-91.

22. Lockhart SR, Reed BD, Pierson CL, Soll DR. Most frequent scenario for recurrent Candida vaginitis is strain maintenance with substrain shuffling: demonstration by sequential DNA fingerprinting with probes Ca3, C1, and CARE2. J Clin Microbiol. 1996;34:767-77.

23. Chong PP, Lee YL, Tan BC, Ng KP. Genetic relatedness of Candida strains isolated from women with vaginal candidiasis in Malaysia. J Med Microbiol. 2003;52:657-66.

24. Alczuk SS, Bonfim-Mendonça PS, Rocha-Brischiliari SC, Shinobu-Mesquita CS, Martins HP, Gimenes F, et al. Effect of highly active antiretroviral therapy on vaginal Candida spp. isolation in HIV-infected compared to HIV-uninfected women. Rev Inst Med Trop Sao Paulo. 2015;57:169-74.

25. Brown AJ, Budge S, Kaloriti D, Tillmann A, Jacobsen MD, Yin $\mathrm{Z}$, et al. Stress adaptation in a pathogenic fungus. J Exp Biol. 2014;217:144-55.

26. Sobel JD. Vulvovaginal candidiasis: a comparison of HIV-positive and -negative women. Int J STD AIDS. 2002;13:358-62.

27. Naglik JR, Rodgers CA, Shirlaw PJ, Dobbie JL, Fernandes-Naglik LL, Greenspan D, et al. Differential expression of Candida albicans secreted aspartyl proteinase and phospholipase $\mathrm{B}$ genes in humans correlates with active oral and vaginal infections. J Infect Dis. 2003;188:469-79.

28. De Bernardis F, Chiani P, Ciccozzi M, Pellegrini G, Ceddia T, D’Offizzi G, et al. Elevated aspartic proteinase secretion and experimental pathogenicity of Candida albicans isolates from oral cavities of subjects infected with human immunodeficiency virus. Infect Immun. 1996;64:466-71.
29. Gruber A, Speth C, Lukasser-Vogl E, Zangerle R, Borg-von Zepelin M, Dierich MP, et al. Human immunodeficiency virus type 1 protease inhibitor attenuates Candida albicans virulence properties in vitro. Immunopharmacology. 1999;41:227-34.

30. Korting HC, Schaller M, Eder G, Hamm G, Böhmer U, Hube B. Effects of the human immunodeficiency virus (HIV) proteinase inhibitors saquinavir and indinavir on in vitro activities of secreted aspartyl proteinases of Candida albicans isolates from HIV-infected patients. Antimicrob Agents Chemother. 1999;43:2038-42.

31. Skrbec D, Romeo D. Inhibition of Candida albicans secreted aspartic protease by a novel series of peptidomimetics, also active on the HIV-1 protease. Biochem Biophys Res Commun. 2002;297:1350-3.

32. Klotz SA, Drutz DJ, Zajic JE. Factors governing adherence of Candida species to plastic surfaces. Infect Immun. 1985;50:97101.

33. Samaranayake LP, MacFarlane TW, editors. Oral candidosis. London: Wright; 1990.

34. Zhao X, Oh SH, Yeater KM, Hoyer LL. Analysis of the Candida albicans Als $2 p$ and Als $4 p$ adhesins suggests the potential for compensatory function within the Als family. Microbiology. 2005;151:1619-30.

35. Paiva LC, Donatti L, Patussi EV, Svizdinski TI, Lopes-Consolaro ME. Scanning electron and confocal scanning laser microscopy imaging of the ultrastructure and viability of vaginal Candida albicans and non-albicans species adhered to an intrauterine contraceptive device. Microsc Microanal. 2010;16:537-49.

36. Van Tornout M, Dadamio J, Coucke W, Quirynen M. Tongue coating: related factors. J Clin Periodontol. 2013;40:180-5.

37. Lian C, Zhao J, Zhang Z, Liu W. Genotype of Candida species associated with different conditions of vulvovaginal candidosis. Mycoses. 2004;47:495-502.

38. Lederman MM, Funderburg NT, Sekaly RP, Klatt NR, Hunt PW. Residual immune dysregulation syndrome in treated HIV infection. Adv Immunol. 2013;119:51-83. 